\title{
Détermination expérimentale des caractéristiques hydrodynamiques d'un sol gonflant non saturé pour la modélisation des écoulements
}

\author{
Experimental determination of hydrodynamic characteristics \\ of a unsaturated-swelling soil for flux modelisation
}

\author{
R. ANGULO, J.-P. GAUDET, J.-L. THONY, M. VAUCLIN \\ Laboratoire d'Etude des Transferts en Hydrologie et Environnement ${ }^{*}$
}

Rev. Franç. Géotech. n 62, pp. 49.57 (janvier 1993)

\section{Résumé}

Une méthode est proposée pour déterminer la conductivité hydraulique et la pression capillaire, en fonction de la concentration en eau, de milieux poreux partiellement saturés susceptibles de se déformer. Elle est fondée sur la description eulérienne des transferts d'eau et de particules solides. Elle s'appuie sur l'analyse en régime transitoire des profils d'humidité et de masse volumique sèche obtenus par spectrométrie gamma double-sources, couplée à des mesures de pression capillaire par tensiométrie. Les résultats présentés sont relatifs à une expérience d'infiltration verticale d'eau dans un matériau poreux compacté et gonflant librement. Le bon accord, entre les valeurs déterminées par l'approche lagrangienne, également mise en œuvre, et celles obtenues dans le cadre de la description eulérienne confirme la pertinence des hypothèses sous-tendant cette dernière. De plus, elle apparaît séduisante dans la mesure où elle peut constituer une modélisation générale des écoulements d'eau, incluant les milieux déformables et rigides.

\footnotetext{
Abstract

A method aimed at determining the hydraulic conductivity and capillary pressure of deformable porous media, as a function of its water content, is proposed. It uses the Eulerian description of the water and solid particles movements. It is based on the analysis of transient volumetric water content and dry bulk density profiles obtained by dual gamma ray technique coupled with measurements of capillary pressure by tensiometers. The results which are presented deal with experiments of vertical infiltration in swelling materials. Fair agreement between the values estimated through the Lagrangian approach which is also used and those obtained in the framework of the Eulerian description tends to demonstrate the validity of the assumptions made to establish the theory developed in the space coordinate at rest. This appears very attractive in the sense that it may be used as the general model of the water flow which includes both deformable and rigid systems.
} 


\section{INTRODUCTION}

Tous les matériaux lorsqu'ils sont soumis à une variation de leur état de contraintes, subissent une déformation plus ou moins significative. Pour les sols, en particulier, cette déformation, induite par des changements de contraintes de type mécanique ou hydraulique, est plus importante que pour beaucoup d'autres matériaux (bétons, roches, métaux, par exemple). De plus, elle évolue à des échelles de temps telles que le praticien, concepteur d'ouvrages (géomécanicien, agronome,...) n'est pas, souvent, en mesure de les prévoir.

Comme pour les milieux à matrice rigide, la connaissance des relations phénoménologiques: conductivité hydraulique - pression capillaire - teneur en eau, constitue un préalable indispensable à toute quantification par voíe expérimentale et de modélisation, des écoulements dans les milieux susceptibles de se déformer (FREDLUND et MORGENSTERN, 1977; LLORET et ALONSO, 1980 ; ALONSO et al., 1990). C'est notamment le cas lorsqu'il s'agit d'estimer le risque de pollution au voisinage des sites souterrains de stockage de déchets, d'étudier la réponse d'un talus aux cycles drainage - humidification, de prévoir la déformation d'un noyau de barrage lors de sa construction et lors de son fonctionnement.

On présente ici une méthode de détermination de ces relations fondée sur lanalyse, dans le cadre d'une description eulérienne des transferts d'eau et de particules solides, de mesures non destructives et simultanées des variations spatio-temporelles d'humidité et de masse volumique sèche, obtenues par la spectrométrie gamma double-sources couplée à des mesures de pression capillaire par la tensiométrie. La démarche proposée est appliquée à un essai d'infiltration dans un échantillon de sol remanié compacté en gonflement libre. A titre indicatif, les résultats sont comparés à ceux quí seraient obtenus par l'approche lagrangienne d'utilisation plus courante en science du sol.

\section{DESCRIPTION EULÉRIENNE ET LAGRANGIENNE DES TRANSFERTS}

\subsection{Description eulérienne des transferts}

Dans l'hypothèse où l'écoulement d'air dans le milieu poreux est instantané, les mouvements des phases liquide et solide, considérés monodirectionnels, sont modélisés, à l'échelle macroscopique, par les deux équations de continuité suivantes:

$$
\begin{aligned}
& \partial \theta_{w} / \partial \mathrm{t}=-\partial \mathrm{q}_{\mathrm{w} / \mathrm{o}} / \partial \mathrm{z} \\
& \partial \theta_{\mathrm{s}} / \partial \mathrm{t}=-\partial \mathrm{q}_{\mathrm{s} / \mathrm{o}} / \partial \mathrm{z}
\end{aligned}
$$

où $\theta_{w}$ et $\theta_{s}=p_{d} / p_{s}=(1+e)^{-1}$ sont respectivement les teneurs volumiques en eau et en particules solides, $e$ est lindice de vides, $\rho_{\text {f }}$ et $p$, les masses volumiques du milieu $\mathrm{sec}$ et du solide; $\mathrm{q}_{\mathrm{w} / \mathrm{o}}$ et $\mathrm{q}_{\mathrm{s} / \mathrm{a}}$ sont les flux volumiques des phases liquide et solide, définis par rapport à un référentiel fixe, celui de l'observateur (axe vertical, $\mathrm{Oz}$, orienté positivement vers le bas). Ils sont liés par la loi de composition des vitesses:

$$
\mathrm{q}_{\mathrm{w} / \mathrm{o}}=\mathrm{q}_{\mathrm{w} / \mathrm{s}}+\mathrm{q}_{\mathrm{s} / \mathrm{o}} \theta_{\mathrm{w}} / \theta_{\mathrm{s}}
$$

où $\mathrm{q}_{\mathrm{w} / \mathrm{e}}$ est le flux d'eau donné par la loi de Darcy exprimée par rapport à la phase solide:

$$
\mathrm{q}_{w / s}=-\mathrm{K}_{w / \mathrm{s}} \partial \Psi_{w} / \partial \mathrm{z}
$$

dans laquelle $\Psi_{w}$ est le potentiel matriciel, exprimé en terme de hauteur de colonne de liquide, et $\mathrm{K}_{\mathrm{w} / \mathrm{s}}$ la conductivité hydraulique que l'on cherche à déterminer. On notera que l'effet de la pesanteur est négligé dans l'équation (3).

Faisons les hypothèses suivantes:

a) Le flux de particules solides est décrit par une loi phénoménologique de transport, de type (YONG et WARKENTIN, 1975 ; NAKANO et al., 1986) :

$$
\mathrm{q}_{\mathrm{s} / \mathrm{o}}=-\mathrm{K}_{\mathrm{s} / \mathrm{o}} \partial \Psi_{\mathrm{s}} / \partial \mathrm{z}
$$

qui peut également s'écrire:

$$
\mathrm{q}_{\mathrm{s} / \mathrm{o}}=-\mathrm{D}_{\mathrm{s} / \mathrm{0}} \partial \theta_{\mathrm{s}} / \partial \mathrm{z}
$$

avec : $\quad \mathrm{D}_{\mathrm{s} / \mathrm{o}}=\mathrm{K}_{\mathrm{s} / \mathrm{o}} \mathrm{d} \Psi_{\mathrm{s}} / \mathrm{d} \theta_{\mathrm{s}}$

où $\Psi_{\text {s }}$ est le potentiel dont le gradient est responsable du mouvement des particules; $K_{s / 0}$ et $D_{s / 0}$ les équivalents, pour la phase solide, de la conductivité hydrau. lique et de la diffusivité capillaire pour la phase liquide.

b) Les potentiels $\Psi_{w}$ et $\Psi_{s}$ sont liés par:

$$
\partial \Psi, \partial z=-v \partial \Psi_{w} / \partial z
$$

avec: $0 \leqslant \nu \leqslant 1$

où v est un paramètre empirique, égal à l'unité pour le milieu saturé (YONG, 1973).

Dans la description du flux de particules solides, $\Psi$ représente le potentiel de pression interne de la phase solide, traduisant l'action et la réaction entre celle-ci et la phase liquide lors du développement de l'écoulement fluide (ANGULO, 1989). Il dépend de la teneur volumique en particules solides, $\theta_{5}$ (ou l'indice de vides, $e$, comme le supposent YONG et WARKENTIN (1975)). Ce potentiel correspond, par exemple, au potentiel de gonflement lorsque la contrainte appliquée au milieu est nulle; si le sol est saturé: $\Psi=-\Psi_{w}$ (PHILIP, 1969; GROENEVELT et BOLT, 1972). Dans ce cas, $\Psi$ a le même sens que la contrainte effective en génie civil, ou que la pression sur des solides compressibles en génie chimique (KIRBY et SMILES, 1988).

Sous ces hypothèses, on peut montrer que l'équation (2) devient:

$$
\mathrm{q}_{\mathrm{w} / \mathrm{o}}=-\mathrm{K}_{\mathrm{w} / \mathrm{o}} \partial \Psi_{w} / \partial \mathrm{z}=-\mathrm{D}_{\mathrm{w} / \mathrm{o}} \partial \theta_{w} / \partial \mathrm{z}
$$

où $\mathrm{K}_{w / \mathrm{o}}$ et $\mathrm{D}_{w / \mathrm{o}}$, définis par:

$$
\begin{aligned}
& \mathrm{K}_{w / 0}=\mathrm{K}_{w / s}-\mathrm{K}_{\mathrm{s} / \mathrm{o}} \nu \theta_{\mathrm{w}} / \theta_{\mathrm{s}} \\
& \mathrm{D}_{w / 0}=\mathrm{K}_{w / 0} \mathrm{~d} \Psi / \mathrm{d} \theta_{w / 0}
\end{aligned}
$$

sont des coefficients apparents de conductivité et de diffusivité de la phase liquide. 
La définition des diffusivités $D_{s / a}$ (équation (4b)) et $\mathrm{D}_{\mathrm{w} / \mathrm{o}}$ (équation $(7 \mathrm{~b})$ ) conduit à :

$$
\mathrm{D}_{\mathrm{s} / \mathrm{o}} / \mathrm{D}_{\mathrm{w} / \mathrm{o}}=-\left(\mathrm{K}_{\mathrm{s} / \mathrm{o}} / \mathrm{K}_{\mathrm{w} / \mathrm{o}}\right) \vee \mathrm{d} \theta_{\mathrm{s}} / \mathrm{d} \theta_{\mathrm{w}}
$$

et l'élimination de $\mathrm{K}_{\mathrm{s} / \mathrm{o}}$ y entre les équations (7a) et (8) amène à:

$$
K_{w / s}=K_{w / o}\left\{1-\left(\theta_{w} / \theta_{s}\right)\left(D_{s / 0} / D_{w / o}\right) d \theta_{1} / d \theta_{w}\right\}
$$

L'introduction des équations (6) et (4b) dans (1a) et (1b) respectivement, conduit au système suivant:

$$
\begin{aligned}
& \partial \theta_{w} / \partial \mathrm{t}=\partial\left\{\mathrm{D}_{w / 0} \partial \theta_{w} / \partial \mathrm{z}\right\} / \partial \mathrm{z} \\
& \partial \theta_{s} / \partial \mathrm{t}=\partial\left\{\mathrm{D}_{\mathrm{w} / \mathrm{o}} \partial \theta_{s} / \partial \mathrm{z}\right\} / \partial \mathrm{z}
\end{aligned}
$$

qui, associé à des conditions initiales et aux limites spécifiques au problème considéré, décrit les mouvements simultanés de l'eau et des particules solides, par rapport au référentiel de l'observateur. On notera que ces deux équations, de type diffusion non linéaire, sont analogues à l'équation de Fokker-Plank couramment utilisée pour modéliser l'écoulement isotherme de l'eau dans un milieu poreux partiellement saturé, à matrice rigide (VAUCLIN et HAVERKAMP, $1985 a$, b; TOUMA et VAUCLIN, 1986). Ainsi, les méthodes de résolution, quasi-analytiques ou numériques, seront les mêmes que le système soit déformable ou non.

\subsection{Description lagrangienne des transferts}

Il est d'usage beaucoup plus courant d'utiliser une description lagrangienne de l'écoulement de l'eau (PHILIP, 1969), dont le principe est brièvement rappelé ci-dessous, puisqu'elle est également mise en œuvre.

L'introduction d'un système de coordonnées matérielles, défini par:

$$
\mathrm{dm} / \mathrm{d} z=(1+e)^{-1}=\theta_{s}
$$

et l'utilisation de lindice d'humidité $v=(1+e) \theta_{w}$ comme variable descriptive de l'état hydrique du milieu, conduit à décrire le transfert d'eau par l'équation de continuité suivante:

$$
\partial u / \partial t=\partial\left\{D_{m} \partial v / \partial m\right\} / \partial m
$$

où la diffusivité " matérielle, $\mathrm{D}_{\mathrm{m}}$, est définie (PHILIP, 1969) par l'expression :

$$
\mathrm{D}_{\mathrm{m}}=\mathrm{K}_{\mathrm{w} / \mathrm{s}}(1+e)^{-1} \mathrm{~d} \Psi_{w} / \mathrm{d} u
$$

qui, compte tenu de la définition de la variable $u$, peut s'écrire :

$$
\begin{aligned}
D_{\mathrm{m}}= & {\left[\mathrm{K}_{\mathrm{w} / \mathrm{s}} \theta_{\mathrm{s}}{ }^{2} \mathrm{~d} \Psi_{\mathrm{w}} / \mathrm{d} \theta_{\mathrm{w}}\right] } \\
& {\left[1-\left(\theta_{\mathrm{w}} \theta_{\mathrm{s}}^{-1}\right) \mathrm{d} \theta_{\mathrm{s}} / \mathrm{d} \theta_{\mathrm{w}}\right]^{-1} }
\end{aligned}
$$

La relation (13b) montre qu'à partir des mêmes données de base que celles précédemment envisagées, la détermination de $\mathrm{K}_{\mathrm{w} / \mathrm{s}}$ nécessite, de plus, la connaissance de $D_{m}$ qui peut être obtenue par la résolution de l'équation (12). En effet, pour des conditions spécifiques, initiale (profil $v(z, 0)=v$, uniforme) et aux limites (condition de Dirichlet, $\left.v(0, t)=v_{1}\right)$, imposées à l'une des faces du milieu poreux, l'utilisation de la variable de Boltzmann (YONG et WARKENTIN, 1975), $\lambda_{\mathrm{m}}=$ $\mathrm{m} \mathrm{t}^{-1 / 2}$, conduit à transformer l'équation (12) en une équation différentielle ordinaire dont l'intégration sur le segment $\left[u_{0}, u\right]$ conduit à :

$$
D_{m}=-(1 / 2)\left(d \lambda_{m} / d u\right) \int_{0}^{u} \lambda_{m} d u
$$

On notera que ce mode de description du transfert d'eau, contrairement au précédent, ne nécessite évidem. ment aucune hypothèse sur la nature du transfert de la phase solide. Les valeurs de la conductivité hydraulique, $\mathrm{K}_{\mathrm{w} / \mathrm{s}}$, ainsi obtenues (éq. (13b)), peuvent donc être utilisées comme références dans la comparaison avec celles prédites par l'équation (9); afin d'apprécier la pertinence des hypothèses (4a) et (5) de lapproche eulérienne.

\section{PROCÉDURE EXPÉRIMENTALE}

Une expérience d'infiltration a été réalisée dans un mélange de bentonite ( $20 \%$ en masse) et de limon compacté dans une cellule en altuglass (diamètre intérieur: $6 \mathrm{~cm}$, hauteur: $6 \mathrm{~cm}$ ), et reposant sur une grille métallique. Pour ce sol on $\mathrm{a}: \mathrm{w}_{\mathrm{f}}=88 \%, \mathrm{w}_{\mathrm{p}}=28 \%$ $(\mathrm{IP}=60)$. La face supérieure de l'échantillon (hauteur initiale: $3 \mathrm{~cm}$ ) a été soumise à une infiltration, par l'in. termédiaire d'une plaque poreuse montée sur un piston en téflon, évidé, servant également de réservoir (fig. 1), le tout étant soumis à une charge hydraulique imposée par un vase de Mariotte. Le déplacement du piston est mesuré par un comparateur.

Au cours de l'infiltration, les évolutions spatio-temporelles de la teneur volumique en eau et de la masse

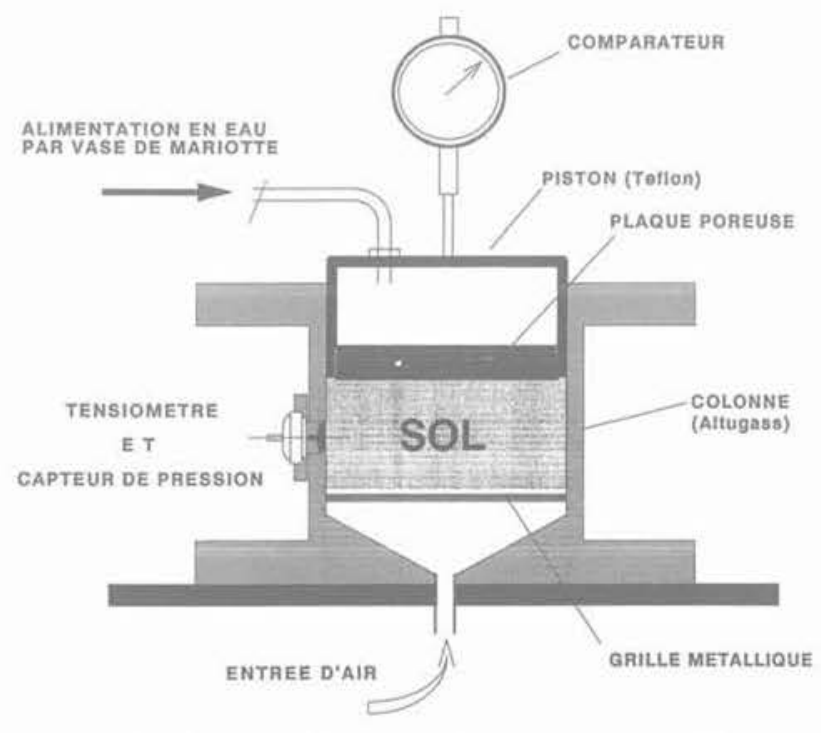

Fig. 1. - Cellule de gonflement et dispositif tensio-capteur.

Fig. 1. - Swelling cell and tensiometer probe. 
volumique sèche sont obtenues au pas $\Delta z=0,5 \mathrm{~cm}$, par deux sources radioactives, ${ }^{137} \mathrm{Cs}$ et ${ }^{241} \mathrm{Am}$, colinéaires, montées sur une plate-forme mobile, portant également le détecteur. La pression de l'eau dans le sol est mesurée par un tensiomètre pariétal implanté à $1,5 \mathrm{~cm}$ du bas de la cellule et connecté à un capteur de pression différentielle. L'ensemble du dispositif est piloté par micro-ordinateur, qui assure également l'acquisition des données brutes (taux de comptages, différences de potentiel électrique, impulsions du moteur) et les transforme en données physiques (teneur volumique en eau $\theta_{w}$, masse volumique sèche $\rho_{d}$, pression tensiométrique $\tau_{w}$, température du détecteur, position $z$ de la plate-forme, temps t depuis le début de l'expérience). Le pas de temps d'acquisition des données a été fixé à 3 heures. La figure 2 présente une vue sché. matique de l'ensemble du dispositif expérimental dé. veloppé.

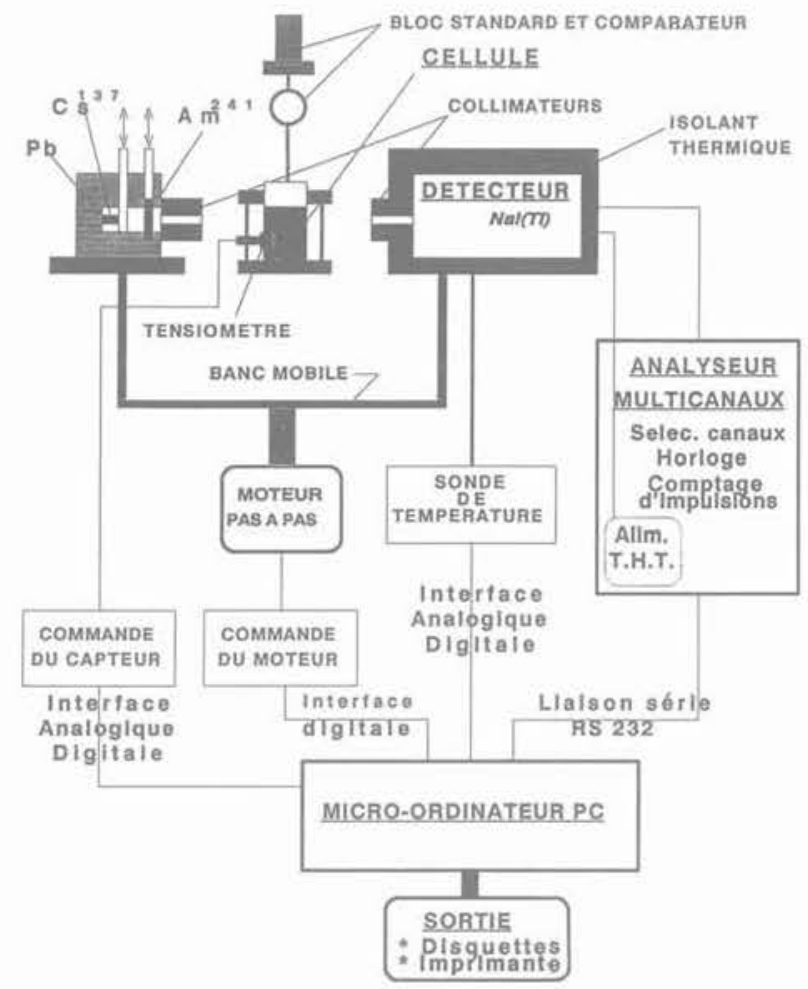

Fig. 2. - Schéma de montage du banc gamma double-sources.

Fig. 2. - A schematic view of the gamma double-ray system.

L'utilisation simultanée des deux sources, jointe à des mesures préalables de l'intensité des faisceaux, I en l'absence de sol dans le porte-échantillon, conduit à deux relations du type:

$$
I_{j}=1_{s} \exp \left\{-X\left(p_{d} \mu_{s}+p_{w} \theta_{w} \mu_{w p}\right)\right\} j=1,2
$$

à deux inconnues $\rho_{\mathrm{d}}$ (masse volumique sèche) et $\theta_{\mathrm{w}}$ (teneur volumique en eau). Dans cette expression, $X$ est l'épaisseur du sol, $\mu_{\mathrm{ss}}$ et $\mu_{\mathrm{w}}$, les coefficients d'absorption massiques pour le solide et l'eau vis-à-vis des deux sources.
La détermination simultanée de $\rho_{\text {p }}$ et $\theta_{\text {p }}$ est obtenue par la résolution du système d'équations linéaires (15a) intégrées sur $t_{c}$ (temps de comptage). Soit:

$$
\left[\begin{array}{l}
P_{d} \\
P_{w} \theta_{w}
\end{array}\right]=(\mathrm{KX})^{-1}\left[\begin{array}{c}
\mu_{w}{ }^{c}-\mu_{w}{ }^{A m} \\
-\mu_{s}{ }^{C_{s}} \mu_{s}{ }^{A m}
\end{array}\right]\left[\begin{array}{l}
\operatorname{Ln}(N / N)_{A m} \\
\operatorname{Ln}(\mathrm{N} / \mathrm{N})_{C_{a}}
\end{array}\right]
$$

où $\mathrm{N}_{\mathrm{y}}$ et $\mathrm{N}$ sont les taux de comptage à travers le porteéchantillon vide et rempli de matériau, $\mathrm{K}=\left(\mu_{1}{ }^{\mathrm{Am}} \mu_{w}{ }_{\mathrm{c}}\right.$ $\left.-\mu_{s}{ }^{C_{s}} \mu_{w}{ }^{A m}\right)$ le déterminant du système qui est différent de zéro pour les sources radioactives utilisées.

A titre d'exemple, la figure 3 donne: les profils hydriques $(3 a)$, les profils de masse volumique sèche $(3 b)$, mesurés à différents instants, depuis l'état initial : $\theta_{\mathrm{w}}=$ $0,450 \pm 0,034 \mathrm{~cm}^{3} / \mathrm{cm}^{3}, p_{d}=1,447 \pm 0,049 \mathrm{~g} / \mathrm{cm}^{3}$, ainsi que les profils de teneur volumique en particules solides $(3 c)$, calculés par $\theta_{s}(z, t)=\rho_{d}(z, t) / \rho_{s}$, où la masse volumique de la phase solide a été estimée à $\rho$. $=2,65 \mathrm{~g} / \mathrm{cm}^{3}$. Les barres horizontales correspondent ầ l'écart-type théorique maximum associé aux mesures de teneur volumique en eau et de la masse volumique sèche (ANGULO, 1989).

Aussi, à partir d'un profil hydrique initial uniforme, on observe clairement le développement progressif d'un front d'humidification à l'instar des milieux rigides, à l'exception de la surface dont la position évolue dans le temps. Au front d'infiltration, correspond un front de gonflement qui se propage également vers le bas de l'échantillon induisant un flux ascendant de particules solides.

\section{CARACTÉRISATION HYDRODYNAMIQUE DU MILIEU}

\subsection{Courbe de gonflement du sol}

Pour la détermination de la perméabilité de Darcy du matériau, selon les approches eulérienne (équation 9) et lagrangienne (équation 13), il est nécessaire de connaitre la relation qui lie la variation de teneur en particules solides (ou de masse volumique sèche) à celle de la teneur volumique en eau : $\theta_{\mathrm{s}}\left(\theta_{\mathrm{w}}\right)$. C'est la courbe de gonflement-retrait pour le chemin de déformation suivi. La gammamétrie double-sources donne un accès direct à cette relation. En effet, pour l'essai d'infiltration considéré, elle a été obtenue par la mise en corrélation entre les valeurs $\theta_{\text {et }} \theta_{s}=\rho_{4} / \rho$. Pour chaque cote de mesure, il est donc possible d'établir une telle relation (fig. 4a).

La figure $4 \mathrm{~b}$ reprend les données de la figure $4 \mathrm{a}$ pour représenter, de façon plus classique, la courbe de gonflement en termes de volume spécifique $\left(v_{-}=1 / \rho_{d}\right)$, en fonction de l'humidité pondérale (w\%). Pour l'essai considéré, elle met en évidence les phases de gonflement normal et résiduel (McGARRY et MALAFANT, 1987; BRAUDEAU, 1988a, b). La présence de bentonite dans le mélange induit un domaine de gonfle. ment normal étendu dont la position proche de la droite théorique à deux phases indique une phase air quasiment négligeable. 
TENEUR VOLUMIQUE EN EAU, $(\mathrm{cm} 3 / \mathrm{cm} 3)$

MASSE VOLUMIQUE SECHE, $(\mathrm{g} / \mathrm{cm} 3)$
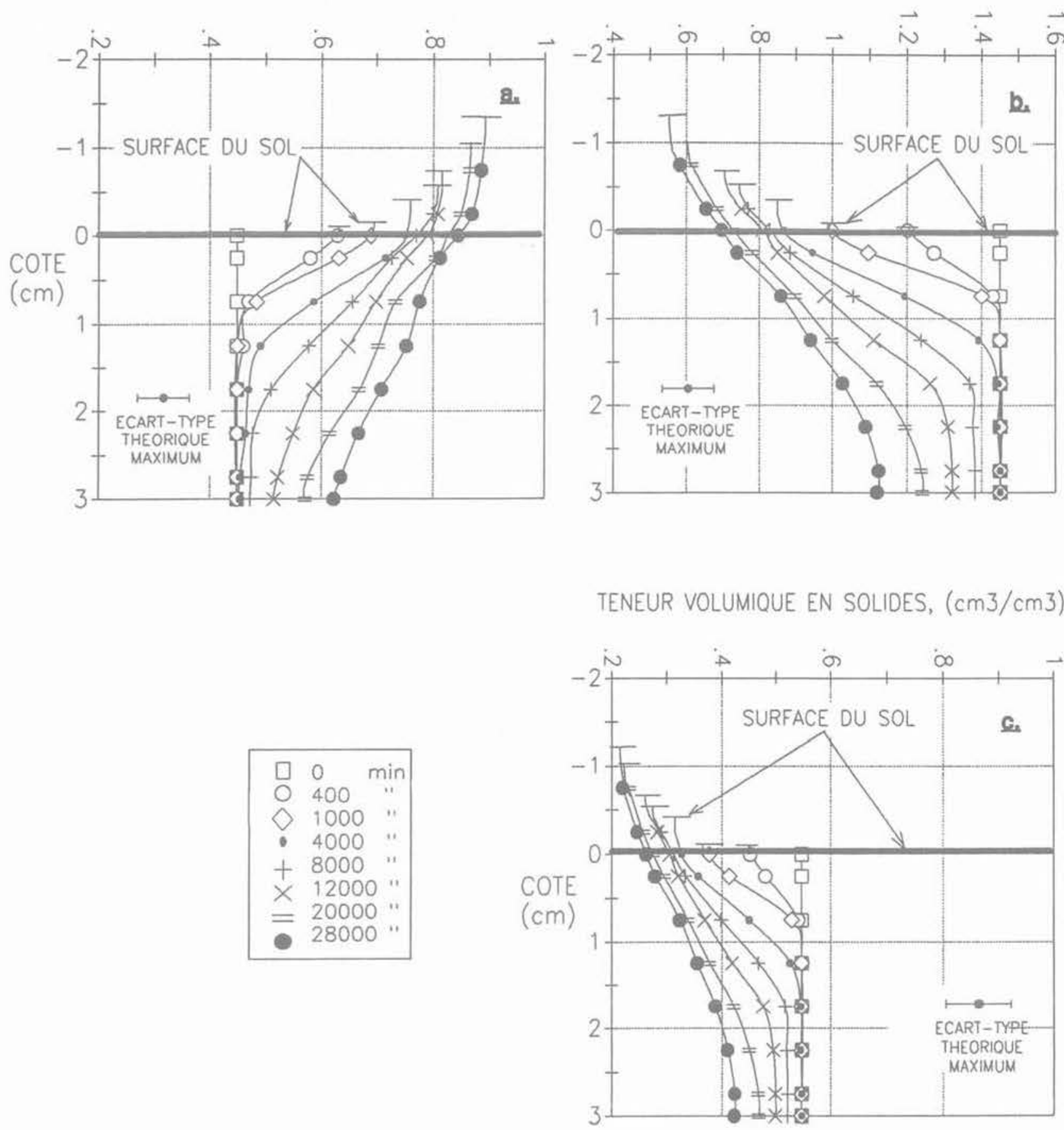

Fig. 3. - Evolution des profils hydriques (a), de masse volumique sèche (b), et de teneur volumique en particules solides (c).

Fig. 3. - Volumetric water content (a), bulk density (b) and volumetric solid particles content (c), profiles.

\subsection{Courbe de rétention d'eau en infiltration}

Les mesures de teneur volumique en eau, effectuées à la cote d'implantation du tensiomètre $(\mathrm{z}=1,5 \mathrm{~cm})$, et les lectures tensiométriques, $\tau_{w}(z, t)$, ainsi que la courbe de gonflement du milieu $\left(\hat{\theta}_{s}\left(\theta_{w}\right)\right.$ ou $\left.\nu_{s}(w)\right)$, permettent d'obtenir la relation entre le potentiel matriciel de l'eau dans le sol et la teneur volumique en eau: $\Psi_{w}\left(\theta_{w}\right)$. Sous l'hypothèse de mise à la pression atmosphérique de l'air du sol, le tensiomètre mesure la somme du potentiel matriciel $\Psi_{u}$ et potentiel de pression (SPOSITO, 1981). Ce dernier dépend du chemin de déformation suivi par le milieu, représenté ici par la courbe de gonflement. 

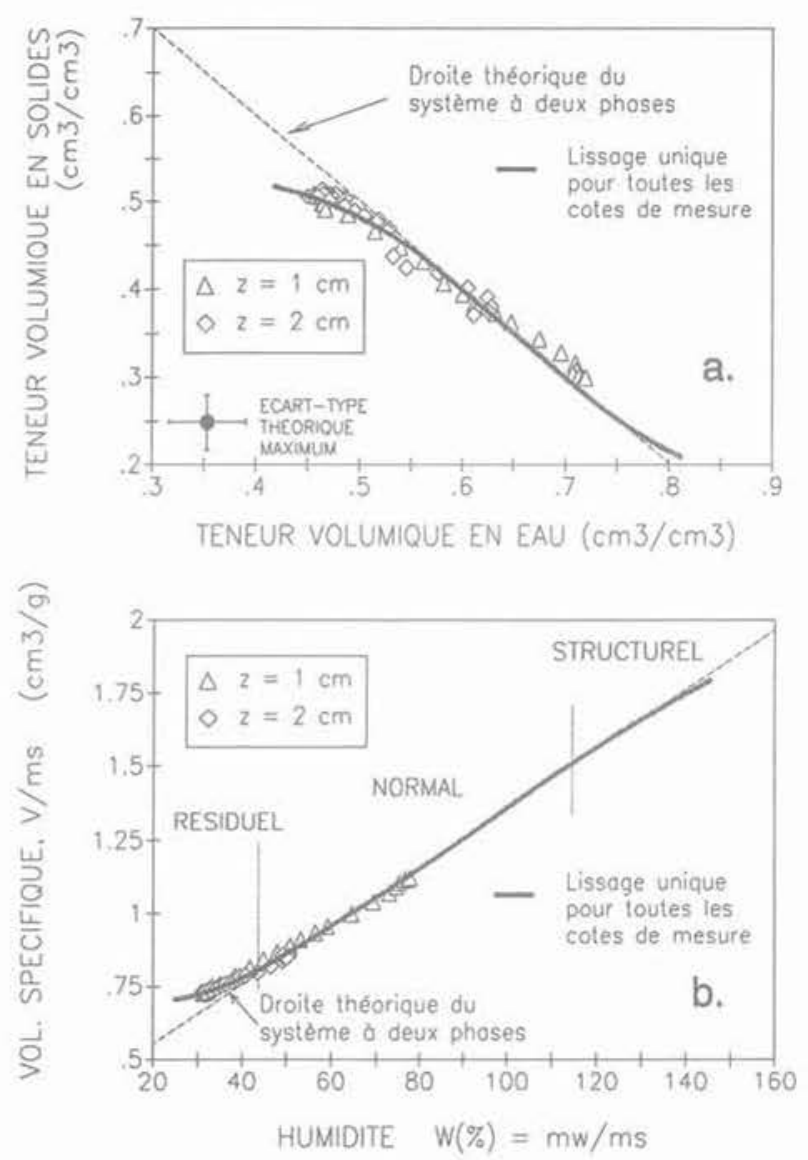

Fig. 4. - Courbe de gonflement: relation entre $\theta_{\mathrm{s}}$ et $\theta_{\mathrm{w}}$ (a), et relation entre $v_{s}=1 / p_{d}$ et $w(b)$.

Fig. 4 - Swelling curve, relationships: between $\theta_{w}$ and $\theta_{s}(a)$, and between $v_{s}=1 / p_{w}$ and $w(b)$.

Le potentiel matriciel, exprimé en termes de hauteur de colonne de liquide est donné par la relation:

$$
\Psi_{w}=\tau_{w} /\left(\rho_{w o} g\right)-\int_{z 0(t)}^{e \tau} \rho \bar{V}_{w} d z
$$

L'intégrale représente la correction à apporter aux lectures tensiométriques due au gonflement, dans laquelle $p=p_{\mathrm{d}}+\rho_{\mathrm{w}} \theta_{\mathrm{w}}$ est la masse volumique du sol surmontant le tensiomètre. $\bar{V}_{w}$ est la pente de la courbe de gonflement (fig. $4 \mathrm{~b}$ ) et $\mathrm{z}_{\mathrm{o}}(\mathrm{t})$ désigne la cote de la surface du sol au temps $t$.

Compte tenu des valeurs relatives à l'essai considéré $\left(\bar{V}_{w}\right.$ voisin de $1 \mathrm{~cm}^{3} / \mathrm{g}, \rho_{d}$ variant de 1,45 à $\left.0,8 \mathrm{~g} / \mathrm{cm}^{3}\right)$, ce terme de correction (vue la faible épaisseur de l'échantillon) varie de 1,8 à $2,5 \mathrm{~cm}$ d'eau. Bien que faible ici, on notera que pour un sol soumis à contrainte (par exemple: essai cedométrique sur sol partiellement saturé), ou pour une mesure tensiométrique in situ, en profondeur, elle peut s'avérer très importante. Par exemple, si la masse volumique moyenne d'un sol gonflant, sur $1 \mathrm{~m}$ de profondeur, vaut $1,8 \mathrm{~g} / \mathrm{cm}^{3}$, et que $\overline{\mathrm{V}}_{\mathrm{w}}=1 \mathrm{~cm}^{3} / \mathrm{g}$, le terme correctif vaudra : $180 \mathrm{~cm}$ d'eau!

La figure 5 présente, pour l'essai considéré la relation $\Psi_{u}\left(\theta_{w}\right)$ qui traduit le pouvoir d'adsorption d'eau du milieu par capillarité.

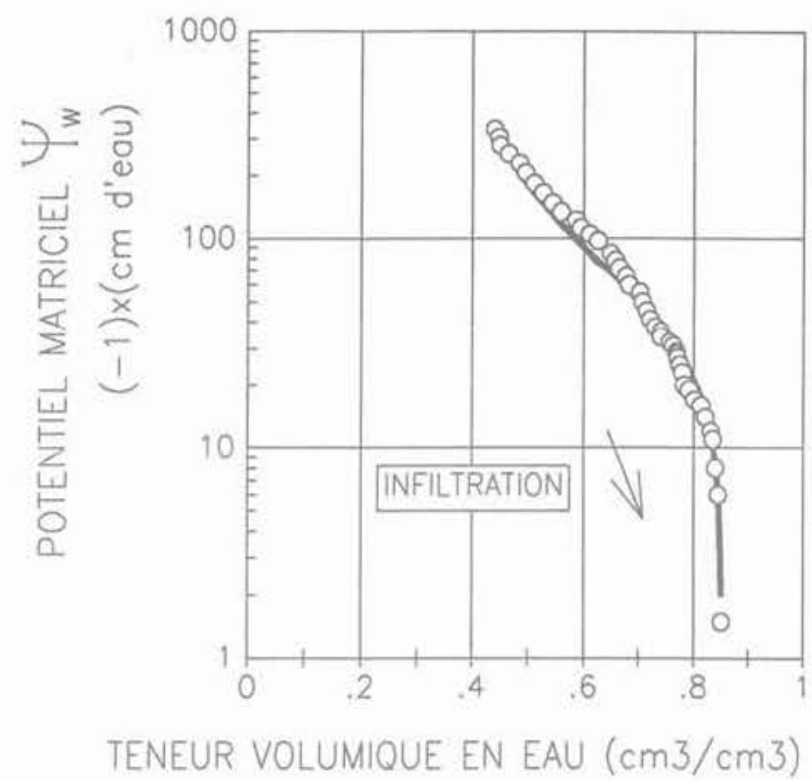

Fig. 5. - Courbe de retention d'eau dans le sol corrigée par effet du gonflement, en fonction de la teneur volumique en eau.

Fig. 5. - Water retention curve of the soil as a fonction of the volumetric water content, corrected for the swell effect.

\subsection{Conductivité hydraulique de Darcy}

La relation (9) permet le calcul de la conductivité hy. draulique, au sens de Darcy, $\mathrm{K}_{\mathrm{w} / \mathrm{s}^{\prime}}$ dès lors que la courbe de gonflement-retrait (fig. 4) et les paramètres apparents $\mathrm{D}_{\mathrm{w} / \mathrm{o}} \mathrm{D}_{\mathrm{s} / 0^{\text {. }}}$ et $\mathrm{K}_{\mathrm{w} / \mathrm{o}}$ sont connus. Compte tenu des conditions initiales et aux limites du problème, l'intégration des équations (10) conduit à l'estimation des flux unitaires apparents d'eau et de particules solides (équations $4 b$ et 6 ) et aux coefficients apparents de diffusivité (fig. 6). Ces derniers sont relatifs au référentiel de mesure et dépendent également du chemin de déformation du milieu. La conductivité apparente est calculée à partir de l'équation $(7 \mathrm{~b})$, de la relation $D_{w / \%}\left(\theta_{w}\right)$ (fig. $6 \mathrm{a}$ ) et de la courbe de rétention $\Psi_{w}\left(\theta_{w}\right)$ (fig. 5).

La figure 7 donne la relation entre $\mathrm{K}_{w / 9} / \mathrm{K}_{w / 0}$, calculée par l'équation (9), et la teneur volumique en eau. On constate que le rapport «Conductivité Darcy/Conductivité Apparente » varie entre 1,5 et 3, environ, dans la gamme des teneurs en eau mesurées. Il apparait donc qu'une erreur du simple au triple peut être commise dans l'estimation de la perméabilité, au sens de Darcy, d'un milieu déformable lorsque l'effet de la déformation de la matrice poreuse n'est pas pris en compte. La figure 8 présente les valeurs de $\mathrm{K}_{\mathrm{w} / \mathrm{s}}$ calculées dans la gamme de teneur volumique en eau explorée et pour le chemin de déformation suivi. Elle présente également les valeurs des conductivités obtenues par lapproche lagrangienne (équation $13 \mathrm{~b}$ ) dans laquelle la diffusivité matérielle $\mathrm{D}_{\mathrm{m}}$ (équation 14 et fig. 9a) et la transformée de Boltzmann $\lambda_{\mathrm{m}}$ (fig. 9b) sont calculées à partir des données de la figure 3 et de la définition de la coordonnée matérielle (équation 11). 


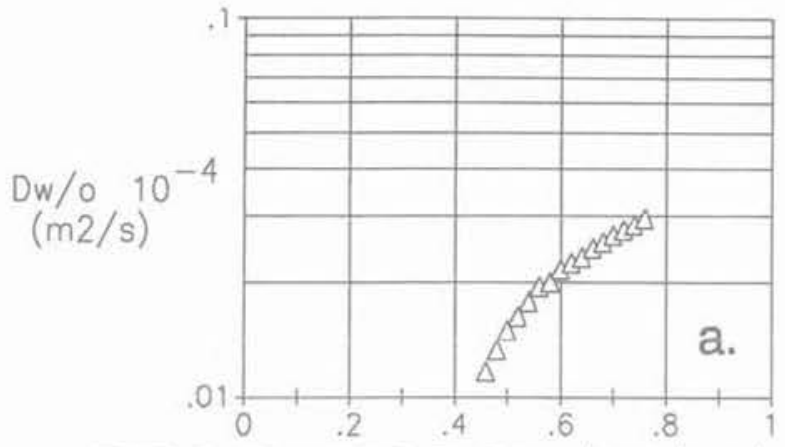

TENEUR VOLUMIQUE EN EAU $(\mathrm{cm} 3 / \mathrm{cm} 3)$

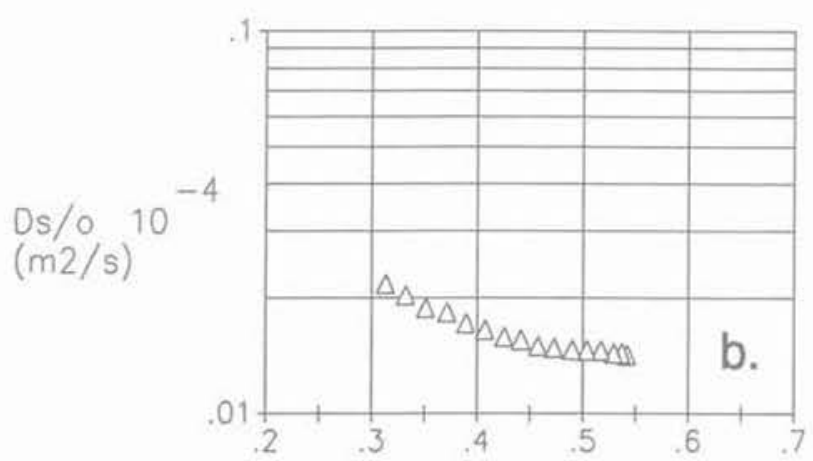

TENEUR VOL. EN SOLIDES $(\mathrm{cm} 3 / \mathrm{cm} 3)$

Fig. 6. - Diffusivité apparente de l'eau (a) et de la phase solide (b) en fonction de la teneur volumique en eau.

Fig. 6. - Apparent diffusivity of water (a) and solid (b) phases as a fonction of volumetric water and solids contents.

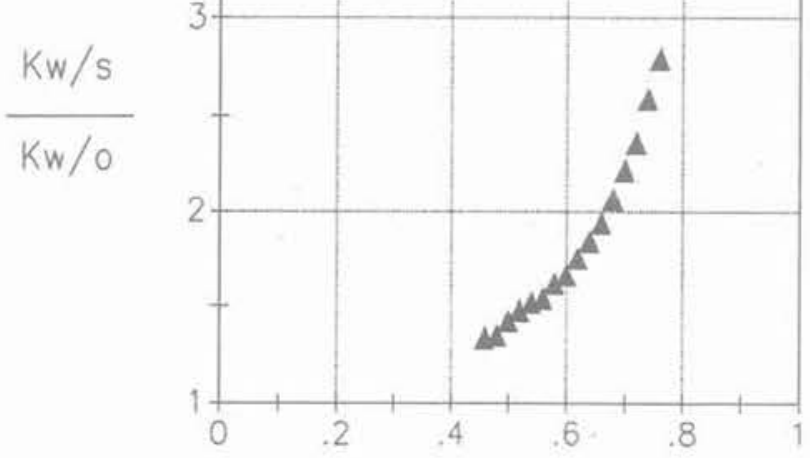

TENEUR VOLUMIQUE EN EAU $(\mathrm{cm} 3 / \mathrm{cm} 3)$

Fig. 7. - Evolution du rapport conductivité de Darcy/ Conductivité apparente, avec la teneur volumique en eau.

Fig. 7. - Evolution of the ratio Darcy Conductivity/. Apparent conductivity as a fonction of volumetric water content.

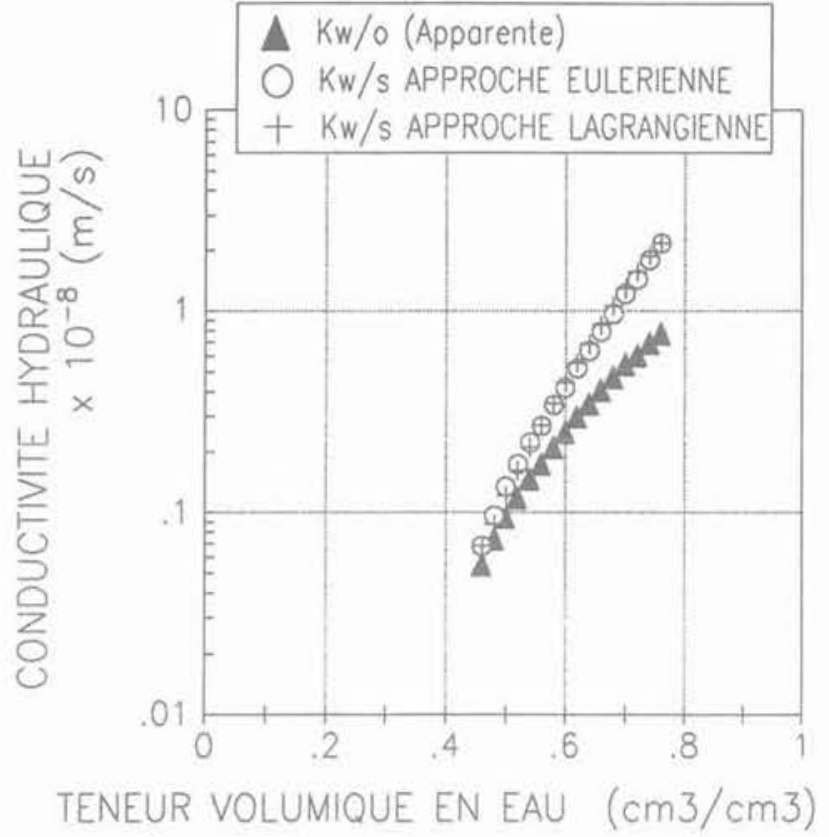

Fig. 8. - Conductivité hydraulique apparente et de Darcy en fonction de la teneur volumique en eau. Comparaison des approches eulérienne et lagrangienne.

Fig. 8. - Hydraulic apparent conductivity and Darcy conductivity as a fonction of volumetric water content. Comparaison between Eulerian and Lagrangian approaches.

\section{CONCLUSION}

L'utilisation d'une métrologie non destructive fondée sur la spectrométrie gamma double-sources et la tensiométrie, couplée à une description eulérienne des trans. ferts, a permis de déterminer, pour un milieu poreux, compacté, partiellement saturé et déformable, les relations conductivité hydraulique - pression capillaire - teneur en eau d'une part, la courbe de gonflement d'autre part, préalable indispensable à toute modélisation du comportement hydromécanique. L'approche eulérienne semble particulièrement pertinente dans la mesure où elle fournit un cadre général d'étude des transferts d'eau dans un milieu poreux partiellement saturé qu'il soit déformable ou rigide.

\section{Remerciements}

La spectrométrie gamma double-sources a été développée et mise au point, grâce à un support financier fourni, pour partie, par le GRECO * Rhéologie des Géomatériaux », groupe Sols Non Saturés.

\section{NOTATIONS}

\section{Concernant la caractérisation du milieu}

$\mathrm{D}_{\mathrm{m}} \quad$ : Diffusivité matérielle

$D_{w / o}^{m} D_{s / o}$ : Diffusivité apparente

$\left[\mathrm{L}^{2} \mathrm{~T}^{-1}\right]$

(par rapport à l'observateur)

$\left[\mathrm{L}^{2} \mathrm{~T}^{-1}\right]$

e $\quad$ : Indice des vides 


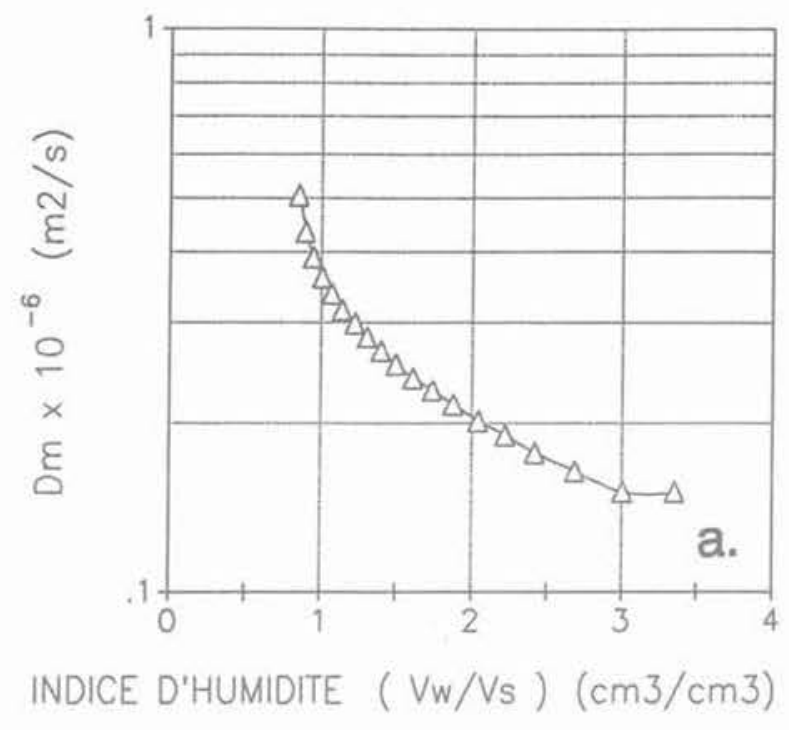

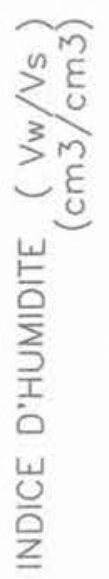

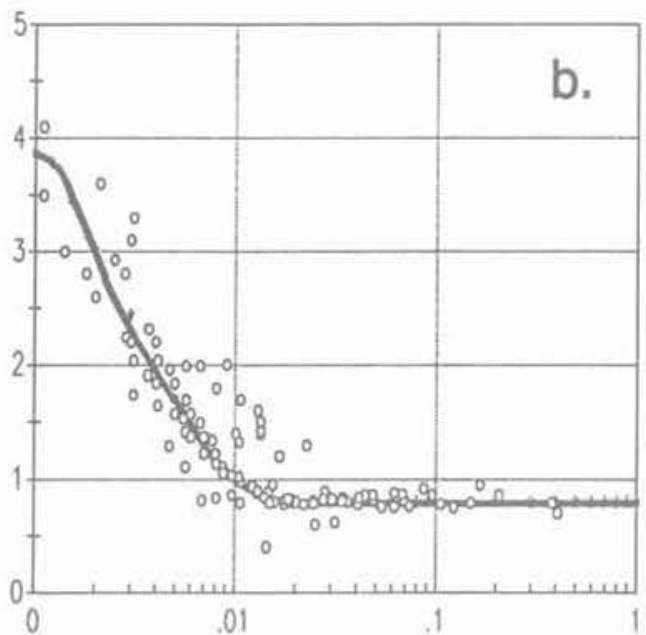

$$
\text { VAR. DE BOLTZMANN } \mathrm{m} / \sqrt{\mathrm{t}}(\mathrm{cm} / \sqrt{\mathrm{min}})
$$

Fig. 9. - Diffusivité matérielle en fonction de l'indice d'humidité (a), profil type indice d'humidité-variable de Boltzmann (b).

Fig. 9. - Material diffusivity as a fonction of humidity ratio (a), relationship between humidity ratio and Boltzmann variable (b)

g : Accélération de la pesanteur $\quad\left[\mathrm{LT}^{-2}\right]$

IP $\quad$ : Indice de plasticité

$\mathrm{K}_{\mathrm{w} / \mathrm{s}} \quad$ : Conductivité hydraulique au sens de Darcy (par rapport à la phase solide) [L $\left.{ }^{-1}\right]$

$\mathrm{K}_{w / 0} \mathrm{~K}_{s / 0}$ : Conductivité hydraulique apparente (par rapport à l'observateur)

$w_{L} \quad$ : Limite de liquidité

$\mathrm{w}_{\mathrm{p}} \quad$ : Limite de plasticité

LR : Limite de retrait

$\mathrm{m} \quad$ : Coordonnée matérielle (phase solide)

$\mathrm{q}_{2 / \mathrm{o}} \mathrm{q}_{\mathrm{a} / \mathrm{s}}$ : Flux volumique, par unité de surface, de la phase $\alpha$ apparent et relatif à la phase solide
$\overline{\mathrm{V}}_{\mathrm{w}} \quad$ : Pente de la courbe

de retrait-gonflement

$\left[\mathrm{L}^{2} \mathrm{~T}^{-2}\right]$

w : Humidité pondérale

$\theta \quad$ : Teneur volumique en phase $\alpha$

u : Indice d'humidité

$\nu_{1} \quad$ : Volume spécifique des solides

$\left[\mathrm{M}^{-1} \mathrm{~L}^{3}\right]$

$v \quad$ : Paramètre de liaison entre les gradients de potentiel des phases solide et liquide

$P_{\alpha} \quad$ : Masse volumique de la phase $\alpha \quad\left[\mathrm{ML}^{-3}\right]$

Pd : Masse volumique sèche $\left[\mathrm{ML}^{-3}\right]$

$\Psi_{\text {w }} \quad$ : Potentiels des phases solide et liquide

$\left[\mathrm{ML}^{-1} \mathrm{~T}^{-2}\right]$

$\lambda_{\mathrm{m}} \quad$ : Variable de Boltzmann matérielle $\left[\mathrm{LT}^{-1 / 2}\right]$

$\tau_{w} \quad$ : Lecture tensiométrique $\quad\left[\mathrm{ML}^{-1} \mathrm{~T}^{-2}\right]$

\section{Concernant la spectrométrie gamma double-sources}

I, I : Intensité de comptage [photons $\mathrm{T}^{-1}$ ]

$\mathrm{K} \quad$ : Déterminant du système d'équations d'absorption du rayonnement gamma double-sources

$\left[\mathrm{M}^{-1} \mathrm{~L}^{4}\right]$

$\mathrm{N}, \mathrm{N}_{\mathrm{v}} \quad$ : Taux de comptage à travers le sol et le vide

[n ${ }^{\circ}$ photons]

$\mathrm{t}_{c} \quad$ : Temps de comptage [T]

$\mathrm{X}$ : Epaisseur d'échantillon [L]

$\mu_{s}, \mu_{w} \quad$ : Coefficient d'absorption massique:

du sol et de l'eau

$\left[\mathrm{M}^{-1} \mathrm{~L}^{4}\right]$

\section{BIBLIOGRAPHIE}

ALONSO E.E., GENS A., JOSA A. (1990), A constitutive model for partially saturated soils. Geotech. nique, 40 (3), pp. 405-430.

ANGULO R. (1989), Caractérisation hydrodynamique de sols déformables partiellement saturés. Etude expérimentale à l'aide de la spectrométrie gamma double-sources. Thèse de Doctorat de l'Institut National Polytechnique de Grenoble (14 décembre 1989).

BRAUDEAU E. (1988), Equation généralisée des courbes de retrait d'échantillons de sol structurés. C. R. Acad. Sci., Paris, 307, Série II, pp. 1731 . 1734.

BRAUDEAU E. (1988), Essai de caractérisation quantitative de l'état structural d'un sol basé sur l'étude de la courbe de retrait. C. R. Acad. Sci. Paris, 307, Série II, pp. 1933-1936.

FREDLUND D.G., MORGENSTERN N.R. (1977), Stress state variables for unsaturated soils. J. Geotech. Eng. Div., ASCE, GT 5, pp. 447-465.

LLORET A., ALONSO E.E. (1980), Consolidation of unsaturated soils including swelling and collapse behavior. Géotechnique, 30 (4), pp. 449-447.

KIRBY J.M., SMILES D.E. (1988), Hydraulic conductivity of acqueous bentonite suspensions. Aust. J. of Soils Res., 26, pp. 561-574.

GROENEVELT P.H., BOLT G.H. (1972), Water retention in soil. Soil Sci., 113 (4), pp. 238-245. 
McGARRY D., MALAFANT K.W.J. (1987), The analysis of volume change in unconfined units of soil. Soil Sci. Soc. Am. J., 51, pp. 290-297.

NAKANO M., AMEMIYA Y., FUJII K. (1986), Satured and unsaturated hydraulic conductivity of swelling soils. Soil Sci., 141 (1), pp. 1-6.

TOUMA J., VAUCLIN M. (1986), Experimental and Numerical Analysis of two-phase infiltration in a partially saturated soil. Transport in Porous Media, 1, pp. 27-55.

VAUCLIN M., HAVERKAMP R. (1985a), Solutions quasi-analytiques de l'équation d'absorption de l'eau par les sols non saturés. I: Analyse critique. Agronomie, 5 (7), pp. 597-606.

VAUCLIN M. HAVERKAMP R. (1985b), Solutions quasi-analytiques de l'équation d'absorption de l'eau par les sols non saturés. II: Problème inverse: détermination de la diffusivité capillaire. Agronomie, 5 (7), pp. 607-611.

PHIL.IP J.R. (1969), Hydrostatics and hydrodynamics in swelling soils. Water Resour. Res., 5 (5), pp. 10701077.

SPOSITO G. (1981), The thermodynamics of soils solutions. Ed. Oxford Clarendon Press.

YONG R.N. (1973), On the physics of unsaturated flow in expansive soils. Proc. 3rd. Int. Conf. on Expansive Soils, Haifa, 2, pp. 1-9.

YONG R.N., WARKENTIN B.P. (1975), Soil properties and behavior. Ed. Elsevier Sci. Pub. Co., Amsterdam, $449 \mathrm{p}$. 